\title{
Multiexon deletions account for $15 \%$ of Congenital Myasthenic Syndrome with RAPSN mutations after negative DNA Sequencing
}

Karen Gaudon, ${ }^{1}$ Isabelle Pénisson-Besnier, ${ }^{2}$ Brigitte Chabrol, ${ }^{3}$ Françoise Bouhour, ${ }^{4}$ Laurence Demay, ${ }^{1}$ Asma Ben Ammar, ${ }^{5,6}$ Stéphanie Bauché, ${ }^{5,7}$ Christophe Vial, ${ }^{4}$ Guillaume Nicolas, ${ }^{2}$ Bruno Eymard, ${ }^{5,7,8}$ Daniel Hantaï , $^{5,8, *}$ Pascale Richard ${ }^{1,9, *}$

${ }^{1}$ AP-HP, UF Cardiogénétique et Myogénétique, Service de Biochimie Métabolique, GH Pitié Salpêtrière, Paris, France

${ }^{2}$ Centre de référence Maladies Neuromusculaires Nantes-Angers, Département de Neurologie, CHU Angers, France

${ }^{3}$ Service de Neuropédiatrie, Hôpital La Timone-Enfants, Marseille, France;

${ }^{4}$ Hôpital Neurologique, GH Lyon Est, France

${ }^{5}$ Inserm, U975, CRICM, GH Pitié Salpêtrière, Paris, France;

${ }^{6}$ INN, La Rabta, Université Tunis El Manar, Tunis, Tunisia;

${ }^{7}$ UPMC, Université Pierre et Marie Curie, Paris

${ }^{8}$ AP-HP, Centre de Référence en Pathologie Neuromusculaire Paris-Est, GH Pitié Salpêtrière, Paris, France;

${ }^{9}$ Inserm, U956, UPMC, Université Pierre et Marie Curie, Paris, France.

* co-last authors

\section{Correspondence should be addressed to:}

Dr. Daniel Hantaï

phone: +33140778145

fax: +331407781 17

e-mail: daniel.hantai@upmc.fr

\section{Total Word Count}

Manuscript: 996 words 
Post-synaptic congenital myasthenic syndromes (CMSs) (OMIM_\#608931) is a group of genetic disorders affecting neuromuscular transmission and due to acetylcholine receptor (AChR) deficiency in $80 \%$ of cases.[1] These autosomal recessive CMSs may be caused by mutations in genes encoding the AChR or one of the AChR-clustering or anchoring proteins, rapsyn, Dok-7 or MuSK.[1-4] Spectra of rapsyn mutations show allelic heterogeneity and suggest that the common substitution p.Asn88Lys (N88K) (variant_021217 in Q13702) results in less stable AChR clusters.[5] Until recently, all patients harbouring mutations in RAPSN are either homozygous for the p.Asn88Lys substitution or heteroallelic for p.Asn88Lys and a mutation which is in most of cases an amino acid substitution but can be also a null allele.[6] Analysis of disease severity in patients suggested that the second mutant allele may largely determine severity of the phenotype.[7] Recently, a patient with two non p.Asn88Lys in RAPSN has been described and the first chromosomal deletion event was described by Müller and colleagues. [8,9]

When analysing 20 patients presenting recessive CMS for RAPSN mutations three out of them were carriers of different large (multi-exonic) but partial deletions of RAPSN that could not be identified by gene sequencing.

Patient 1 is a 22-year-old girl presenting a severe generalized hypotonia at birth with arthrogryposis, retrognathism and amimic face with no sucking reflex. There was no respiratory problem or dysphagia. She achieved independent walking on tiptoes at 16 months and presented several episodes during which she was unable to walk. At age 17, she showed a mild proximal muscle weakness in the four limbs and in neck flexors, a reduced mouth opening and a slight limitation in left eye abduction. EMG demonstrated a clear decrement on 3-Hz repetitive stimulation in trapezius and quadriceps muscles.

Patient 2 is a 27 -year-old woman presenting a severe generalized hypotonia at birth with arthrogryposis of the ankles, knees, elbows, and fingers. She had facial diplegia, respiratory 
failure with weak cries, no suction, no swallowing, and a permanent defect of orbicular muscles of lips and eyes. The diagnosis of CMS was confirmed after a negative test for AChR antibodies, a $15 \%$ decrement and a positive prostigmine test.

Patient 3 is a 7-years-old girl with no family history. At birth, a major hypotonia and arthrogryposis of the hands and feet were noted. She was not able to swallow and had frequent respiratory failure episodes leading to mechanical ventilation. The prostigmine test was positive and the EMG showed a decrement confirming the diagnosis of CMS.

Molecular analyses revealed in Patient 1, an "apparently" homozygous RAPSN substitution (c.264c>a), p.Asn88Lys transmitted by the unaffected mother but not carried by the father suggesting a "missed" variant not detected by usual sequencing approach. In both Patients 2 and 3, the missense p.Asn88Lys substitution was identified on a single allele with no identification of the second allelic mutation. In both families, heterozygous parent for p.Asn88Lys was the mother. We determined the RAPSN copy number in each patient, using three SNPs known to cosegregate (rs7111873, rs34729771 and rs7126210; fig 1). In these three patients, the SNPs do not cosegregate correctly. In patient 1, rs34729771 and rs7126210 SNPs were heterozygous while rs7111873 was homozygous. In patient 2, only the SNPs rs7111873 was heterozygous. In patient 3, only rs7111873 and rs34729771 were heterozygous. A relative quantification for each exon of RAPSN was performed by a qPCR approach. In patient 1, a deletion involving 5'UTR, promoter, exon 1 and exon 2 was detected (fig 2). Partial delimitation of breakpoints showed that the deletion corresponding to a $\sim 30 \mathrm{~kb}$ occurred between rs 2242081 and intron 2. In patient 2, allelic quantification confirms the loss of exon 3 to 7 on one allele and the long range PCR/sequencing showed that the recombination occurred between a short sequence of 7 nucleotides (CCTGCAG) in intron 2 at the junction with exon 3 (c.532-7; c.532-1) and the same sequence at the 5' end of exon 8 (c.1170_1176) resulting in a deletion spanning on $4.785 \mathrm{~kb}\left(\mathrm{~g} .47,416,165 \_47,420,949 \mathrm{del}\right)$. In patient 3, the deletion involves exon 7 to 8 and part of 
3'UTR and the recombination occurred between two sequences of 25 nucleotides

(GCTAATTTTTGTATTTTTAGTAGAG), the first located in intron 6 (c.967-398_967-374) and the other one located in 3'UTR region resulting in a deletion of $10.334 \mathrm{~kb}(\mathrm{~g}$.

47,417,456_47,407,123del). The allele quantification was performed in the parents and showed that in the 3 families, the chromosomal micro-deletion was transmitted by the father (for experimental details see Supplemental data files 1 and 2).

In our series of CMS patients recruited via the French CMS National Network, twenty patients were found with disease-causing mutations in RAPSN. Among these patients, three (15\%) had the recurrent p.Asn88Lys substitution but the sequencing approach failed to identify the second allelic mutation. We hypothesized that genomic deletions may account in these patients and developed a simple molecular assay based on qPCR analysis. This led us to identify three different chromosomal micro-deletions due to recombinations. All these multi-exon deletions corresponded to the missing disease-causing allelic mutation in patients.

The description of Müller and colleagues and ours demonstrates that RAPSN, containing numerous repeated sequences, is particularly subject to multiple genomic recombinations. The precise deletions breakpoints determination showed that the recombination involves multiple and different sequences in all the described cases.

Altogether these findings lead to the following comments: (i) a negative result obtained by direct sequencing of genomic DNA raises the question of an incomplete detection of some mutations; (ii) in recessive transmissions, the analysis of patient parents is crucial to confirm the inheritance; (iii) phenotype-genotype correlations should also be considered:while patient 1 did not exhibit a very severe phenotype during childhood, patients 2 and 3 presented a CMS with all criteria of severity corresponding to the phenotype observed in patients carrying the p.Asn88Lys substitution associated with another allelic mutation.[10] 
In conclusion, this mutational mechanism represents $15 \%$ of patients with RAPSN mutations referred to our laboratory. For diagnostic purposes, carrier detection, genetic counselling, and prenatal diagnosis, it is critical to know the exact functional gene copy number that an individual carries.

Key Words: Congenital myasthenic syndrome, rapsyn, chromosomic microdeletion, loss of heterozygosity, allele copy number.

\section{Acknowledgements}

The authors would like to thank the patients and their families for their helpful collaboration. This work was supported by Assistance Publique-Hôpitaux de Paris (PHRC AOM 1036), Réseaux Inserm, ANR-Maladies Rares (ANR-07-MRAR-001), Association Française contre les Myopathies and a Contrat d'Interface AP-HP Inserm (to DH).

Competing Interests: None to declare.

\section{Copyright licence statement}

The Corresponding Author has the right to grant on behalf of all authors and does grant on behalf of all authors, an exclusive licence (or non exclusive for government employees) on a worldwide basis to the BMJ Publishing Group Ltd to permit this article (if accepted) to be published in Journal of Medical Genetics and any other BMJPGL products and sublicences such use and exploit all subsidiary rights, as set out in our licence (http://group.bmj.com/products/journals/instructions-for-authors/licenceforms)." 


\section{REFERENCES}

1. Engel AG, Sine SM. Current understanding of congenital myasthenic syndromes. Curr Opin Pharmacol 2005;5:308-21.

2. Ohno K, Engel AG, Shen XM, et al. Rapsyn mutations in humans cause endplate acetylcholinereceptor deficiency and myasthenic syndrome. Am J Hum Genet 2002;70:875-85.

3. Chevessier F, Faraut B, Ravel-Chapuis A, et al. MUSK, a new target for mutations causing congenital myasthenic syndrome. Hum Mol Genet 2004;13:3229-40.

4. Beeson D, Higuchi O, Palace J, et al. Dok-7 mutations underlie a neuromuscular junction synaptopathy. Science 2006;313:1975-8.

5. Cossins J, Burke G, Maxwell S, et al. Diverse molecular mechanisms involved in AChR deficiency due to rapsyn mutations. Brain 2006;129:2773-83.

6. Banwell BL, Ohno K, Sieb JP, Engel AG. Novel truncating RAPSN mutations causing congenital myasthenic syndrome responsive to 3,4-diaminopyridine. Neuromuscul Disord 2004;14:202-7.

7. Ioos C, Barois A, Richard $\mathrm{P}$, et al. Congenital myasthenic syndrome due to rapsyn deficiency: three cases with arthrogryposis and bulbar symptoms. Neuropediatrics 2004;35:246-9.

8. Maselli RA, Dris H, Schnier J, et al. Congenital myasthenic syndrome caused by two non-N88K rapsyn mutations. Clin Genet 2007;72:63-5.

9. Müller JS, Abicht A, Christen HJ, et al. A newly identified chromosomal microdeletion of the rapsyn gene causes a congenital myasthenic syndrome. Neuromuscul Disord 2004;14:744-9.

10. Burke G, Cossins J, Maxwell S, et al. Rapsyn mutations in hereditary myasthenia: distinct earlyand late-onset phenotypes. Neurology 2003;61:826-8. 


\section{Figure legends:}

Figure 1: Schematic representation of RAPSN. Exons are numbered from 1 to 8 . The common p.Asn88Lys substitution and the three known allelic SNPs are indicated by arrows. Presence or absence of these polymorphisms for the three patients is indicated: presence of the SNP heterozygous (+); absence of the SNP (-).

Figure 2: Graphic representation of the relative quantification of RAPSN exons. Results were expressed in $\mathrm{N}$-fold changes in RAPSN exon copies, normalized to $\beta$-globin relative to the copy number of the target gene. When $0.8<\mathrm{N}$-fold $<1.7$, the DNA sample harboured two copies of the RAPSN exon. If $\mathrm{N}$-fold was $<0.7$, the sample harboured only one copy of the exon. qPCR in patients revealed a clear reduction to about $50 \%$ for the deleted exons, exon 1 and 2 in patient 1 , exons 3 to 7 in patient 2 and exons 7 and 8 in patient 3 . 


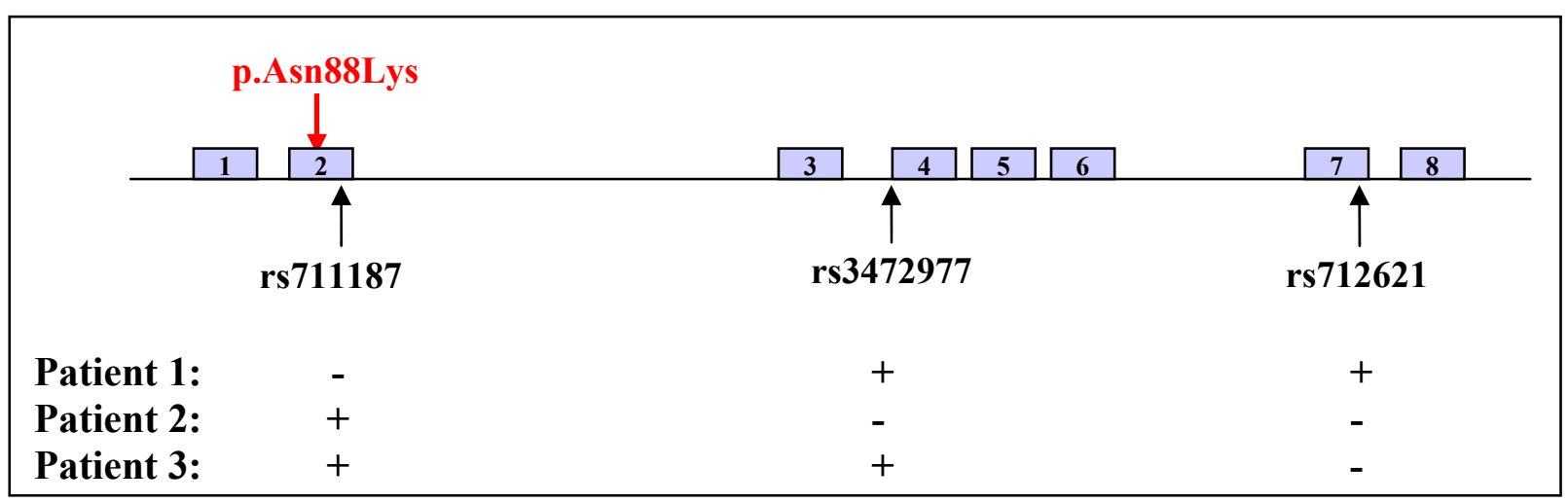




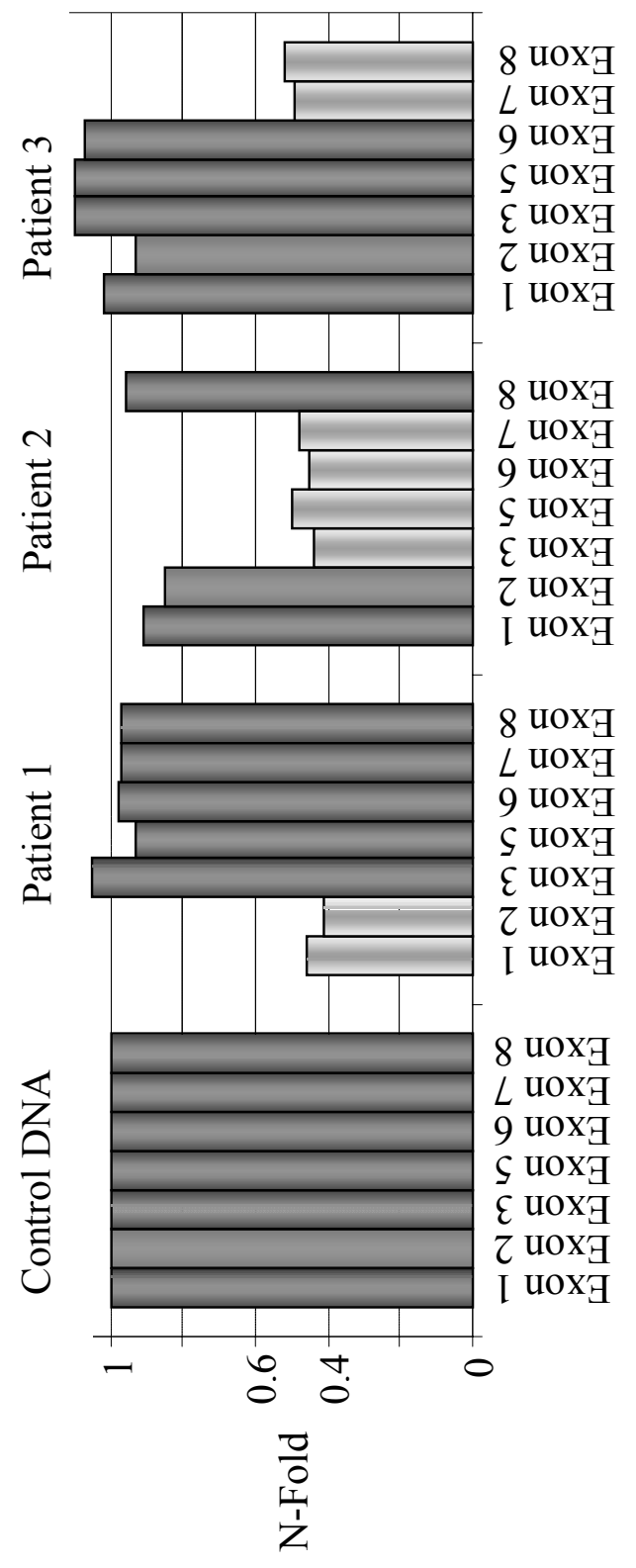

